\title{
A CLASS OF CURVES ON WHICH POLYNOMIALS APPROXIMATE EFFICIENTLY ${ }^{1}$
}

\author{
D. J. NEWMAN AND L. RAYMON
}

A normal curve is defined to be a continuous curve of finite length contained in the unit square $0 \leqq x, y \leqq 1$. It is well known that the uniform closure of the polynomials on a normal curve, $Q$, is the class of all functions continuous on $Q$. In this note we concern ourselves with the accuracy of the approximation of continuous functions on $Q$ by $n$th degree polynomials. In particular, we seek a class of normal curves on which the accuracy of the approximation by $n$th degree polynomials compares favorably with that of any other $n^{2}$-dimensional set of approximating functions. In order to state our result precisely, we make the following definitions:

Let $Q$ be a compact set in $E^{k}$ (Euclidean $k$-space). The massivity, $m_{n}(Q)$, is a sequence defined as follows: Let $X_{n}$ be a set of $n+1$ elements of $Q$; then

$$
m_{n}(Q)=\max _{X_{n} \subset Q} \min _{x_{i}, x_{j} \in X_{n} ; i \neq j}\left|x_{i}-x_{j}\right| .
$$

Given a function $f(x)$ defined on $Q$ and a $\delta>0$, the modulus of continuity of the function $f(x)$ is defined as usual:

$$
\omega_{f}(\delta)=\sup _{|x-y| \leqq \delta}|f(x)-f(y)| .
$$

If $\omega_{f}(\delta) \leqq \delta$ for all $\delta>0, f(x)$ is said to be a contraction on $Q$. Let $C(Q)$ be the space of all real valued continuous functions on $Q$ and let $P$ be a finite dimensional subspace of $C(Q)$. Let $K$ denote the class of contractions on $Q$. We define the "degree of approximation" as

$$
\rho(P)=\max _{f \in K} \min _{p \in P} \max _{x \in Q}|f-p| .
$$

An $n$th degree polynomial in more than one variable is defined to be a polynomial in which the maximum degree in any single variable is less than $n$. Unless otherwise specified, $P$ will denote the subspace consisting of $n$th degree polynomials. In this case $\rho(P)$ may be denoted $\rho_{n}$.

Jackson's classical theorem established that if $Q$ is the interval $0 \leqq x \leqq 1$, then there exist positive constants $c_{1}, c_{2}$ such that

Received by the editors March 6, 1967.

1 This research was partially supported by the National Science Foundation, Grant No. NSF GP-4391. 


$$
c_{1} / n \leqq \rho_{n} \leqq c_{2} / n \quad[2, \text { p. } 14] .
$$

It is also known that if $Q$ is a normal curve then $\rho_{n}=O(1 / n)[4$, Lemma 5], while for $P$ any $n^{2}$-dimensional subspace of $C(Q)$,

$$
\rho(P) \geqq m_{n^{2}} / 2 \geqq c / n^{2} \quad[3],[4, \text { Lemma } 1] .
$$

Thus, if $Q$ is a curve for which the degree of approximation is equal to $O\left(1 / n^{2}\right)$, polynomials can be termed efficient on $Q$. We now state

THEOREM 1. Let $Q$ be the curve $y=\sum_{k=1}^{\infty} a_{k} x^{k}, 0 \leqq x \leqq 1$, where $\left|a_{1}\right| \leqq 1$ and $\left|a_{n+1}\right|<\left|a_{n}\right|^{n}$. Then there exist positive constants $c_{1}, c_{2}$ such that

$$
c_{1} / n^{2} \leqq \rho_{n} \leqq c_{2} / n^{2} .
$$

In particular, polynomials are efficient on the curve given by

$$
y=\sum_{n=1}^{\infty} \frac{x^{n}}{n^{n^{n} \log n}}, \quad 0 \leqq x \leqq 1 .
$$

Leмma. If $\sum_{k=0}^{n} a_{k} x^{k} \leqq 1,0 \leqq x \leqq 1$, then

$$
\left|a_{k}\right| \leqq 2^{2 k} \frac{n(n+k-1) !}{(n-k) !(2 k) !} .
$$

To derive this bound, one demonstrates that

$$
T_{n}(2 x-1)=\sum_{k=0}^{n}(-1)^{k} 2^{2 k} \frac{n(n+k-1) !}{(n-k) !(2 k) !} x^{k}
$$

is maximal for each coefficient. For proof, see $[1$, p. 30]. Note that the above upper bounds yield the estimate $\left|a_{k}\right| \leqq 3^{2 n}, 0 \leqq k \leqq n$.

PROOF OF THE THEOREM. By (1), $\rho_{n} \geqq c_{1} / n^{2}$. To prove that $\rho_{n} \leqq c_{2} / n^{2}$, we shall choose an $n$th degree approximating polynomial in the following manner. Let $f$ be an arbitrary contraction. Let $S_{n}(x)$ be the $n$th partial sum of the infinite series, $S(x)$. Consider $f$ to be extended onto the unit square by setting $f(x, y)=f(x, S(x))$. It will be shown that there is some $n$th degree polynomial, $p_{n}(x, y)$, such that $\left|f-p_{n}\right|$ $\leqq c / n^{2}, c$ independent of $n$. This polynomial will be our approximating polynomial on the given curve. The proof will be completed by showing that when passing from one curve to another along a line parallel to the $y$-axis, the variation of $p_{n}$ is of smaller order than $1 / n^{2}$.

Let $Q_{n}$ be the curve $y=S_{n}(x)$. Any monomial, $x^{k}, k=0,1, \cdots$, $n^{2}+n$, on $Q_{n}$ is equal to an $n$th degree polynomial in $x$ and $y$ as follows: 


$$
\begin{aligned}
x^{k} & =x^{k}, \quad k=0,1, \cdots, n, \\
x^{n+1} & =\frac{x y-\left(a_{1} x^{2}+a_{2} x^{3}+\cdots+a_{n-1} x^{n}\right)}{a_{n}} .
\end{aligned}
$$

Now,

$$
x^{n+k}=\frac{x^{k} y-\left(a_{1} x^{k+1}+a_{2} x^{k+2}+\cdots+a_{n-1} x^{k+n-1}\right)}{a_{n}}, k=1,2, \cdots, n \text {, }
$$

is a polynomial of degree $n$ in $x$ and of first degree in $y$ by induction on $k$. Now,

$$
\begin{array}{r}
x^{m n+k}=\frac{x^{(m-1) n+k} y-\left(a_{1} x^{(m-1) n+k+1}+\cdots+a_{n-1} x^{(m-1) n+k+1}\right)}{a_{n}}, \\
m=1,2, \cdots, n ; k=0,1, \cdots, n,
\end{array}
$$

is a polynomial of degree $n$ in $x$ and of degree $m$ in $y$ by induction on $m$ and $k$.

It follows from the identities (2) that on $Q_{n}$ any polynomial in $x$ of degree $n^{2}, \sum_{0}^{n^{2}} b_{k} x^{k}$, can be expressed as $\sum_{0}^{n} c_{k}(x) y^{k}$, where $c_{k}(x)$ is an $n$th degree polynomial. It is desirable to find an upper bound for $\left|c_{k}(x)\right|, k=0,1, \cdots, n, 0 \leqq x \leqq 1$. From the relations (2), a calculation verifies that

$$
n^{3}\left|\frac{a_{n-1}}{a_{n}}\right|_{k}^{n^{2}} \max _{k}\left|b_{k}\right|
$$

is an upper bound. If $\left|\sum_{0}^{n^{2}} b_{k} x^{k}\right| \leqq A$, we have, by the lemma, and from the inequality

$$
\left|a_{n-1}\right|^{n^{2}}<O\left(1 / n^{7} 3^{2 n^{2}}\right)
$$

the estimate

$$
\max _{k, x}\left|c_{k}(x)\right| \leqq A / 2 n^{4} a_{n}^{n^{2}} .
$$

Now, let $f$ be an arbitrary contraction extended on $Q_{n}$ as described. Let $g(x)=f\left(x, S_{n}(x)\right), 0 \leqq x \leqq 1$. If $\left|g^{\prime}(x)\right|<M$, then $g(x) / M$ is a contraction. (By the hypothesis on $\left|a_{n}\right|, M<1+1 /\left(1-\left|a_{2}\right|\right)$.) Hence, by Jackson's theorem, there is an $n^{2}$-degree polynomial, $p_{n 2}(x)$, such that

$$
\left|g(x)-p_{n^{2}}(x)\right| \leqq M c / n^{2}, \quad 0 \leqq x \leqq 1,
$$

where $c$ is the constant of Jackson's theorem. Let $p_{n}(x, y)$ be the $n$th degree polynomial gotten from $p_{n 2}(x)$ by the relations (2). For any $x \in[0,1]$, 


$$
\begin{aligned}
& \left|f(x, S(x))-p_{n}(x, S(x))\right| \leqq\left|f(x, S(x))-f\left(x, S_{n}(x)\right)\right| \\
& \quad+\left|f\left(x, S_{n}(x)\right)-g(x, 0)\right|+\left|g(x, 0)-p_{n^{2}}(x, 0)\right| \\
& \quad+\left|p_{n^{2}}(x, 0)-p_{n^{2}}\left(x, S_{n}(x)\right)\right|+\left|p_{n^{2}}\left(x, S_{n}(x)\right)-p_{n}\left(x, S_{n}(x)\right)\right| \\
& \quad+\left|p_{n}\left(x, S_{n}(x)\right)-p_{n}(x, S(x))\right| .
\end{aligned}
$$

By Taylor's theorem,

$$
\max _{x \in[0,1]}\left|p_{n}\left(x, S_{n}(x)\right)-p_{n}(x, y)\right| \leqq \max _{x \in[0,1]} \sum_{k}\left|\frac{d^{k}}{d y^{k}} p_{n}(x, y)\left(y-S_{n}(x)\right)^{k}\right| \frac{1}{k !} .
$$

Since, in particular, for $y=S(x)$ we have

$$
\left|y-S_{n}(x)\right|<2\left|a_{n+1}\right| \text {, }
$$

(3) gives us

$$
\left|p_{n}\left(x, S_{n}(x)\right)-p_{n}(x, S(x))\right| \leqq A / n^{2} .
$$

We have already established that the third term on the right-hand side of (4) is bounded by $M c / n^{2}$, while the first, second, fourth and fifth terms are zero by definition. Hence,

$$
\left|f(x, S(x))-p_{n}(x, S(x))\right|<(M c+A) / n^{2} \text {. Q.E.D. }
$$

In Theorem 1, we have considered approximation to the class of contractions, $K$. This theorem will now be extended to any class of functions equicontinuous on $Q$. Let $P$ be defined as before. Let $C$ be the class of all functions in $C(Q)$, whose modulus of continuity is $\leqq \omega(\delta)$ (where $\omega(\delta)$ is the modulus of continuity of some continuous function) and define

$$
\rho^{*}(P)=\max _{\boldsymbol{j} \in C} \min _{\boldsymbol{p} \in P} \max _{\boldsymbol{x} \in \boldsymbol{Q}}|f-p| .
$$

Theorem 2. Let $Q$ be the curve

$$
y=\sum_{k=1}^{\infty} a_{k} x^{k}, \quad 0 \leqq x \leqq 1,
$$

where $\left|a_{n+1}\right|<\left|a_{n}\right|^{n^{2}}$ and $\left|a_{1}\right| \leqq 1$. Then there exist positive constants, $c_{1}, c_{2}$, such that

$$
c_{1} \omega\left(1 / n^{2}\right) \leqq \rho_{n}^{*} \leqq c_{2} \omega\left(1 / n^{2}\right) .
$$

Let $Q$ be a simple normal curve. For $z$, w $\in Q$, denote by $d(z, w)$ the length of that portion of $Q$ connecting $z$ to $w$. We call $Q$ properly normal if there exists $M$ such that $d(z, w) \leqq M|z-w|$.

Theorem 2 follows immediately from Theorem 1 and the following 
Lemma. If $Q$ is a properly normal curve of length 1 , then

$$
\rho_{n}^{*}(P)<4 M \omega\left(\rho_{n}(P)\right) .
$$

Proof. Let $f \in C$. Let $z_{1}$ be an endpoint of $Q$. By hypothesis, $0 \leqq d\left(z_{1}, z\right) \leqq 1$. Partition $Q$ in to $[1 / \rho]$ curves, $Q_{k}, k=1,2, \cdots,[1 / \rho]$, of equal length. Suppose, first, that $1 / \rho$ is an integer. Consider $L(z)$, the function equal to $f(z)$ at the endpoints, $z_{k}, z_{k+1}$, of the $Q_{k}$, and defined "linearly" in between; i.e.,

$$
L(z)=\frac{\left(z-z_{k}\right) f\left(z_{k+1}\right)-\left(z_{k+1}-z\right) f\left(z_{k}\right)}{z_{k+1}-z_{k}}, \quad z \in Q_{k} .
$$

We have, then, $\left|L\left(z_{k+1}\right)-L\left(z_{k}\right)\right| \leqq \omega\left(\left|z_{k+1}-z_{k}\right|\right) \leqq \omega(\rho)$. Hence,

$$
|L(z)-L(w)| \leqq \omega(\rho) L(z, w) / \rho \leqq M \omega(\rho)|z-w| / \rho,
$$

implying that $\rho L(z) / M \omega(\rho)$ is a contraction and, thus, that there exists $p_{1}(z)$ such that

$$
\left|\rho L(z) / M \omega(\rho)-p_{1}(z)\right|<\rho .
$$

Letting $p_{2}(z)=M p_{1}(z) \omega(\rho) / \rho$, we have

$$
\left|L(z)-p_{2}(z)\right| \leqq M \omega(\rho) .
$$

Now,

$$
\begin{aligned}
\left|f(z)-p_{2}(z)\right| \leqq & \left|f(z)-f\left(z_{k}\right)\right|+\left|f\left(z_{k}\right)-L\left(z_{k}\right)\right| \\
& +\left|L\left(z_{k}\right)-L(z)\right|+\left|L(z)-p_{2}(z)\right| \leqq 3 M \omega(\rho) .
\end{aligned}
$$

We take care of the case where $1 / \rho$ is not an integer by weakening our estimate to $4 M \omega(\rho)$.

\section{REFERENCES}

1. S. N. Bernstein, Lȩ̧ons sur les propriétés extrémales et la meilleure approximation des fonctions analytiques d'une variable réelle, Gauthier-Villars, Paris, 1926.

2. D. Jackson, The theory of approximation, Amer. Math. Soc. Colloq. Publ., Vol. 11, Amer. Math. Soc., Providence, R. I., 1930.

3. G. G. Lorentz, Lower bounds for the degree of approximation, Trans. Amer. Math. Soc. 97 (1960), 25-34.

4. D. J. Newman and L. Raymon, Quantitative polynomial approximation on certain planar sets, Trans. Amer. Math. Soc. (to appear).

Yeshiva UNIVERSITY AND

TEMPLE UNiversity 